\title{
TAILORING THE INTERNAL MICROSTRUCTURE OF THE HYDROGELS BASED ON POLY- HEMA TARGETED FOR DRUG DELIVERY SYSTEMS
}

\author{
${ }^{1}$ Monika TRUDICOVA, ${ }^{1}$ Hana PAPEZIKOVA, ${ }^{1}$ Petr SEDLACEK, ${ }^{1}$ Miloslav PEKAR \\ ${ }^{1}$ Brno University of Technology, Faculty of Chemistry, Brno, Czech Republic, EU, \\ xctrudicova@fch.vut.cz
}

https://doi.org/10.37904/nanocon.2021.4350

\begin{abstract}
Transport and barrier properties are important material characteristics in terms of the development of the targeted drug delivery systems. Therefore, this work focused on the study of the possibility of influencing these properties and internal architecture in a model hydrogel system formed by a semi-interpenetrating polymer network with an incorporated polyelectrolyte component. Due to its application potential and its extensive use in medicine, poly(2-hydroxyethyl methacrylate) was chosen as the model hydrogel system for this work. Sodium polystyrene sulfonate was used as the interpenetrating linear polymer. Scanning electron microscopy was used to examine the structure of the three-dimensional hydrogel network. Transport properties were monitored by diffusion tests. Furthermore, diffusion through the hydrogel (barrier properties) was monitored using diffusion cells (Franz cells, horizontal cells). The usability of the semi-interpenetrating polymer network concept for the preparation of materials with tunable relevant properties was confirmed on the proposed materials.
\end{abstract}

Keywords: Hydrogel, poly(2-hydroxyethyl methacrylate), transport properties, drug delivery systems

\section{INTRODUCTION}

Hydrogels; also defined as systems consisting of a three-dimensional network of polymer chains and water filling the space between these macromolecules; have acquired a key position in diverse fields of biomedicine, such as in wound dressings, controlled release, or as scaffolds in tissue engineering. The properties of hydrogels must vary according to the specific needs of individual applications, e.g., for drug delivery systems, suitable transport properties are crucial to their application. Recent studies [1-4] have focused on various strategies to improve these properties in hydrogels using a process such as the use of interpenetrating networks, composite structures, and dual crosslinking. Overall, hydrogel preparation methods that simultaneously mimic the hydration, strength, and stiffness of soft and supporting tissues have the potential to be used in a much wider range of biomedical applications. Using semi-interpenetrating polymer networks (semi-IPN) is a suitable way to modify the material properties by manipulation of the internal chemical and morphological architecture [2-4].

Hydrogels based on poly(2-hydroxyethyl methacrylate) (poly-HEMA) were the first synthetic hydrogels for pharmaceutical applications, they are biologically compatible hydrogels with tunable mechanical properties [5]. They are usually synthesized by the polymerization of methacrylate with free radicals. Due to their properties, poly-HEMA hydrogels are suitable to produce hydrophilic contact lenses and artificial corneas [6]. Poly-HEMA is also an interesting material for drug delivery systems, e.g. chitosan blends with this polymer are commonly used in drug delivery systems [7,8]. For these reasons, poly-HEMA was chosen as the model hydrogel system in this work. 


\section{MATERIALS AND METHODS}

In this work, the synthesis took place by radical polymerization by photoinitiation. All chemicals used in this work were purchased from Sigma Aldrich (97\% 2-hydroxyethyl methacrylate (HEMA) CAS: 868-77-9; 98\%; ethylene glycol dimethacrylate (EGDMA) CAS: 97-90-5; 98\% Irgacure 2959 CAS: 106797-53-9). All components of the solution were mixed in a vial in a ratio of 2:3 by volume deionized water and 2-hydroxyethyl methacrylate monomer, with the addition of EGDMA as a crosslinker and Irgacure 2959 as an initiator. A thin layer of the liquid mixture was poured into a petri dish, then placed under the UV lamp so that the resulting radiation intensity was about $2.4 \mathrm{~mW} / \mathrm{cm}^{2}$. At this radiation intensity, the synthesis time was 30 minutes. The samples containing an incorporated component in the form of a polyelectrolyte were also prepared. Sodium polystyrene sulfonate (PSS, Sigma-Aldrich, CAS: 25704-18-1) was added to the above polymer solution in such an amount that its final concentration in the reaction mixture was $0.1 \mathrm{~g} / \mathrm{l}$. After the solidification of hydrogels, it was necessary to remove a residual monomer. The hydrogels were immersed in ethanol for 24 hours and then in deionized water or only in deionized water. During the immersion in ethanol, changes in structural properties of hydrogels were evident, which were further investigated in the subsequent experimental set.

Determining the structural character of a hydrogel is a crucial part of understanding its transport properties. The literature [5] and previous experiments have shown that upon contact with water (after removing residual monomer), the optical properties of the hydrogel (turbidity) can change significantly, which is related to the change in the internal structure. To determine the change in the internal structure, hydrogels were subjected to direct visualization by scanning electron microscopy (SEM). For SEM observations small parts of the specimens were taken. These specimens were subsequently gold-coated in a sputtering device and investigated using a scanning electron microscope ZEISS EVO LS 10 in the mode of secondary electrons (SE). The accelerating voltage was set to $5 \mathrm{kV}$.

To investigate transport properties, Rhodamine 6G (R6G) was used as a diffusion probe since its structure (aromatic cation) models some real active substances (e.g. analgesics) and the same time allows an easy spectroscopic detection (photometry, fluorimetry). Transport properties were monitored by diffusion tests (absorption of the dye into the internal structure of the gel, subsequent release from the gel). Throughout the experiments, the absorbance of dye solutions was measured at certain times on a UV-VIS spectrometer in the wavelength range from 800 to $300 \mathrm{~nm}$. Furthermore, diffusion through the gel (barrier properties) was monitored using diffusion cells (vertical Franz cells, horizontal side-by-side cells).

\section{RESULTS AND DISCUSSION}

\subsection{Structural analysis}

Freeze-drying using a regular freezer is usually associated with significant structural artifacts or collapse of the internal structure of hydrogels caused by the formation and growth of large ice crystals. On contrary, rapid freezing (e.g. with the use of liquid nitrogen) that supports vitrification can be a way to preserve the internal structure of the hydrogels without artifacts [9]. In this case, the results showed that for a hydrogel containing only about $30 \mathrm{wt} \%$ water, it does not matter whether liquid nitrogen or a freezer is used.

The analysis was performed on a total of six samples. Three with the addition of polyelectrolyte and three without. The first pair of hydrogels was frozen immediately after hydrogel synthesis and is shown in Figure 1. The hydrogel structure is smooth, and no pores are visible. Occasional clusters may indicate some polymers not incorporated into the structure (oligomers) which remained on the surface of the hydrogel. Water content and surface suggest that it is a microporous or even non-porous type of HEMA hydrogel. 

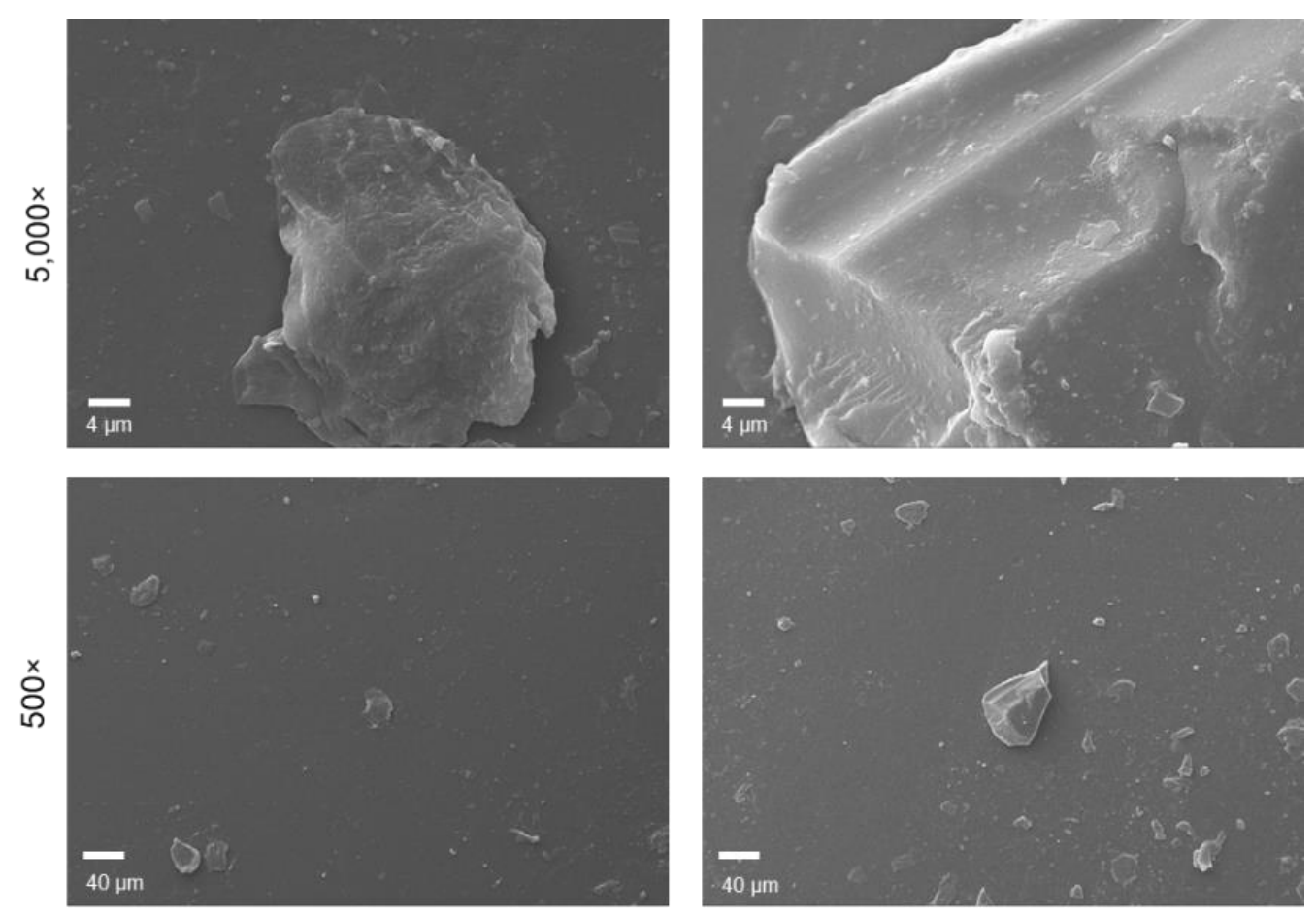

Figure 1 Poly-HEMA hydrogels frozen immediately after synthesis: without (left) and with (right) the addition of PSS

During immersion of the fresh hydrogels in ethanol (while washing the samples to remove the uncrosslinked components), anisotropic swelling of the sample occurred preferentially in the horizontal direction and changes in structural properties (indicated by reduced turbidity) were observed (shown in Figure 2). Therefore, the effect of ethanol on the structure was investigated further. For this purpose, another pair of samples was placed in ethanol for 24 hours before freezing and is shown in Figure 3A. The swelling of the samples can be demonstrated by the wrinkling of the surface of the samples. At a magnification of $5,000 \times$, it could be said that there are small cracks between the individual parts.

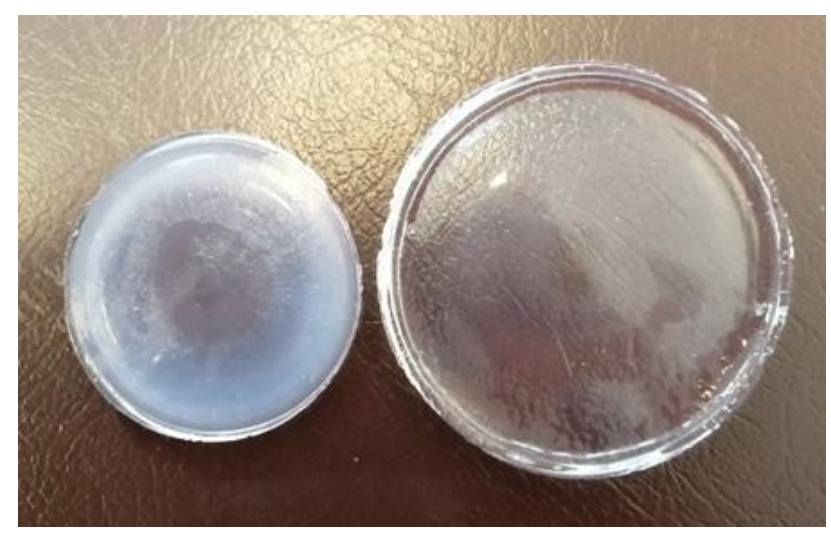

Figure 2 Demonstration of swelling and changes in the structure of the hydrogel (sample with the addition of PSS) after 24 hours in water (left) and after 24 hours in ethanol (right)

The third pair of samples was immersed in ethanol for 24 hours and then in water for 24 hours before freezing and its structure is shown in Figure 3B. Occasional pores to holes are visible in these samples. One of the possible causes is the reduction of the gel volume, where these pores formed from previous cracks. Another possibility may be that during the elution, ethanol has ripped some part of the network out of the structure. 

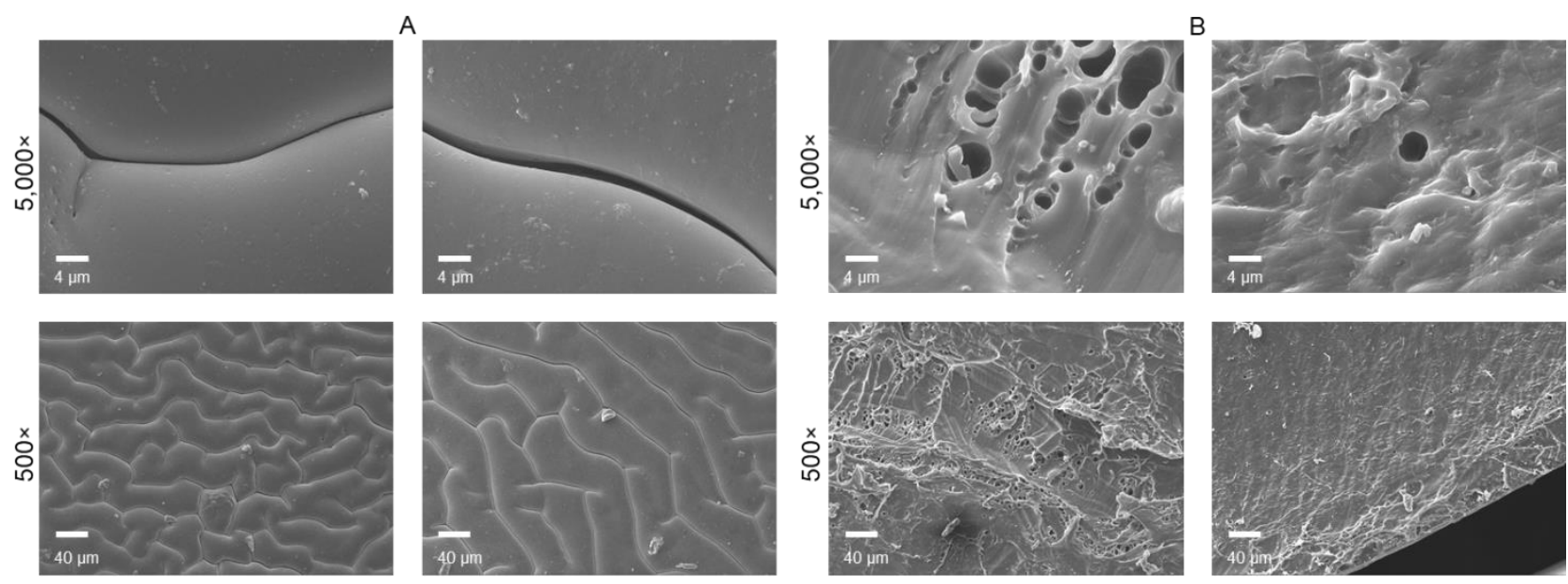

Figure 3 Poly-HEMA hydrogels frozen after 24 hours in ethanol (part A): without (left) and with (right) the addition of PSS and poly-HEMA hydrogels after 24 hours in ethanol and the following 24 hours in the water (part B): without (left) and with (right) the addition of PSS

\subsection{Study of transport properties}

During the first experiment, uptake of the dye from solutions with different concentrations into the internal structure of the hydrogel was monitored. From the attached graph (Figure 4), which shows the average absorbance values at $526 \mathrm{~nm}$ in the dye solution over time, the samples with PSS have a greater ability to absorb the dye solution into the hydrogel structure, most likely due to an electrostatic attraction between the cationic dye and the opposite charge on PSS. Nevertheless, as can also be seen in Figure 4, the initial uptake tendency of the two gels has the opposite trend, i.e. more dye is absorbed by the gel without PSS soon after the start of the experiment. This may be explained by an effect of PSS on the effective porosity and tortuosity of the gel since the addition of polyelectrolyte may reduce the number of diffusion trajectories available for the dye. The final absorbance value is similar in both cases. The result is due to the total rhodamine concentration that was initially chosen. After 24 hours during the experiment, almost all the dye was diffused into the hydrogel. This trend was observed for samples immersed in all prepared rhodamine concentrations.

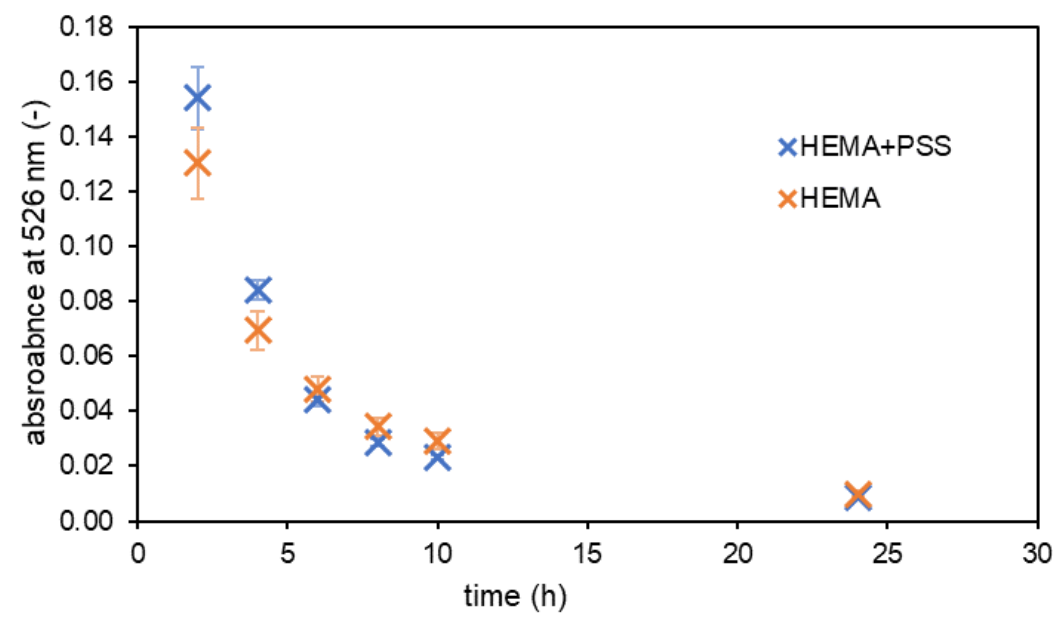

Figure 4 Example of graph for Experiment 1 of absorbance versus experiment time (for immersing in solution with highest rhodamine concentration)

In the second experiment, the release of the dye (after its absorption) from the hydrogel into the environment was monitored. This time, higher concentrations of rhodamine were used than in the previous experiment. 
First, rhodamine is released into the external environment of the hydrogel, but at some point, the hydrogel begins to resorb the dye back into its structure. Another interesting phenomenon observed in this experiment is the fact that PSS not only absorbs more dye but also, interacts with it and the dye remains in the structure, which can be seen in Table 1 (the rhodamine concentration is lower in the hydrogel samples with PSS throughout this part of the experiment).

Table 1 Experiment 2 (release of dye from hydrogels after absorption): rhodamine concentration in water over 72 hours (X-poly-HEMA hydrogel, PSS - with the addition of PSS, 1 - absorption from 0,025 $\mathrm{g} / \mathrm{l} 6 \mathrm{G}, 2$ - absorption from 0,05 g/l R6G and 3 - absorption from 0,1 g/l R6G)

\begin{tabular}{|c|c|c|c|c|c|c|}
\hline & $\mathbf{X}-1$ & PSS-1 & \multicolumn{2}{c|}{$\mathbf{X}-2$} & PSS-2 & \multicolumn{2}{c|}{$\mathbf{X}-3$} & PSS-3 \\
\hline $\mathbf{t}(\mathbf{h})$ & \multicolumn{2}{|c|}{$\mathbf{c}(\boldsymbol{\mu g} / \mathbf{l})$} & \multicolumn{2}{|c|}{$\mathbf{c}(\boldsymbol{\mu g} / \mathbf{l})$} & \multicolumn{2}{c|}{$\mathbf{c}(\boldsymbol{\mu g} / \mathbf{l})$} \\
\hline 12 & 9.434 & 6.261 & 19.26 & 12.56 & 44.22 & 30.24 \\
\hline 24 & 4.937 & 3.705 & 11.62 & 6.970 & 33.33 & 15.82 \\
\hline 36 & 2.566 & 2.904 & 5.430 & 4.213 & 18.72 & 8.802 \\
\hline 48 & 1.811 & 2.088 & 3.459 & 3.305 & 8.356 & 5.445 \\
\hline 60 & 1.826 & 1.996 & 2.612 & 2.859 & 5.784 & 4.737 \\
\hline 72 & 1.765 & 1.749 & 2.134 & 2.412 & 4.306 & 3.782 \\
\hline
\end{tabular}

Next, a series of experiments to monitor the resorption of dye after immersing hydrogel with absorbed dye into the water was performed. The results showed that after the water change every 12 hours, the diffusion of rhodamine happens continuously from the hydrogel into the external environment and no resorption occurs. The results suggest that changes in the arrangement and binding of the polymer chains may occur due to the diffusion of rhodamine.

To prevent the resorption of the transported substance, in the next experiments, hydrogels were prepared directly with rhodamine in their structure (during synthesis). The gels had to be synthesized for 45 minutes instead of 30. A possible explanation is that rhodamine enters the polymerization reaction and forms bonds with free radicals or that it removes UV radiation due to its fluorescence. These experiments again showed the effect of a polyelectrolyte, which retained more dye in the hydrogel than the hydrogel without it.

Finally, another set of experiments was performed to monitor the effect of immersion of the samples in ethanol on the subsequent diffusion. The samples were first immersed for 24 hours in ethanol, then in water to wash thoroughly, and then in the dye solution. For comparison, the experiment was performed with the same concentration of rhodamine in samples without immersion in ethanol. The results showed that the hydrogels immersed in ethanol absorbed far more Rhodamine $6 \mathrm{G}$ during the diffusion of the dye into the internal structure than the samples immersed in water. One possible explanation is that the higher absorption capacity is caused by the pores, which were evident in chapter 2.1., where it was shown that their formation occurs with the use of ethanol. These hydrogels again showed the effect of a polyelectrolyte, which absorbed more dye.

The barrier properties were investigated using diffusion cells, where a solution of the rhodamine dye was present in the donor chamber and pure deionized water was present in the receiving chamber. First, experiments were performed with vertically oriented Franz cells. The hydrogel has a water content of about 30 wt \% and with decreasing water content in the hydrogel, the free volume of hydrogel available for diffusion transport decreases, and the curvature of the diffusion trajectory increases. The first non-zero absorbance in the characteristic spectral region was thus recorded on the 12th day. Next, horizontal diffusion cells were subsequently used for this experiment. Triplicates, three samples of poly-HEMA hydrogel, and three with the addition of PSS were prepared. During the experiment, the average values of the concentrations again showed a higher barrier ability of hydrogels containing polyelectrolyte. 


\section{CONCLUSION}

This work focused on monitoring the influence of an incorporated polyelectrolyte component on the resulting transport properties and internal architecture of a model hydrogel system in terms of the development of the targeted drug delivery systems. The dried samples were subjected to morphological analysis by scanning electron microscopy. The results showed that the freezing process did not affect these hydrogels. The results show that during freezing, the pores are reduced, and the surface of the gels is smooth. During the immersion in ethanol, changes in the structural properties of hydrogels occurred.

Experiments to determine the transport properties have demonstrated the influence of the incorporation of a semi-interpenetrating polyelectrolyte component (PSS). The hydrogels with the addition of PSS were able to absorb a larger amount of diffusion probe (Rhodamine 6G) into their structure. Certainly, the electrostatic interaction of the polyelectrolyte and the dye, which are oppositely charged and therefore attract each other, had a key role. The barrier properties of the hydrogels were investigated using diffusion cells. The influence of the incorporated polyelectrolyte on diffusion through the hydrogel was confirmed.

From the obtained results it can be stated that the use of semi-IPN can modify the transport and barrier properties of the model poly-HEMA hydrogels, which are the key properties for the use of these materials in controlled drug release systems.

\section{REFERENCES}

[1] MEANS, A.K., GRUNLAN, M.A. Modern strategies to achieve tissue-mimetic, mechanically robust hydrogels. ACS Macro Letters. [online]. 2019, vol. 8, no. 6, pp. 705-713. Available from: https://doi.org/10.1021/acsmacrolett.9b00276.

[2] MYUNG, D., WATERS, D., WISEMAN, M., DUHAMEL, P.E., NOOLANDI, J. TA, C.N., FRANK, C.W. Progress in the development of interpenetrating polymer network hydrogels. Polymers for Advanced Technologies. [online]. 2008, vol. 19, no. 6, pp. 647-657. Available from: https://doi.org/10.1002/pat.1134.

[3] TRUDICOVA, M., SMILEK, J., KALINA, M., SMILKOVA, M., ADAMKOVA, K., HRUBANOVA, K., KRZYZANEK, V., SEDLACEK, P. Multiscale experimental evaluation of agarose-based semi-interpenetrating polymer network hydrogels as materials with tunable rheological and transport performance. Polymers. [online]. 2020, vol. 12, no. 11, 2561. Available from: https://doi.org/10.3390/polym12112561.

[4] LIN, C.C., METTERS, A.T. Hydrogels in controlled release formulations: network design and mathematical modeling. Advanced Drug Delivery Reviews. [online]. 2006, vol. 58, no. 12-13, pp. 1379-1408. Available from: https://doi.org/10.1016/j.addr.2006.09.004.

[5] BAKER, M.V., BROWN, D.H., CASADIO, Y.S., CHIRILA, T.V. The preparation of poly(2-hydroxyethyl methacrylate) and poly\{(2-hydroxyethyl methacrylate)-co-[poly(ethylene glycol) methyl ether methacrylate]\} by photoinitiated polymerisation-induced phase separation in water. Polymer. [online]. 2009, vol. 50, no. 25, pp. 5918-5927. Available from: https://doi.org/10.1016/j.polymer.2009.10.047.

[6] ROTARU, I., OLARU, D. Mechanical behaviour of $\mathrm{p}(\mathrm{HEMA})$ hydrogel for disc prosthesis on lumbar spine. Journal of Optoelectronics and Advanced Materials. 2014, vol. 16, no. 7-8, pp. 881-886.

[7] GARCIA, J., RUIZ-DURANTEZ, E., VALDERRUTEN, N.E., Interpenetrating polymer networks hydrogels of chitosan and poly(2-hydroxyethyl methacrylate) for controlled release of quetiapine. Reactive and Functional Polymers. [online]. 2017, vol. 117, pp. 52-59. Available from:

https://doi.org/10.1016/j.reactfunctpolym.2017.06.002.

[8] PEPPAS, N. Hydrogels in pharmaceutical formulations. European Journal of Pharmaceutics and Biopharmaceutics. [online]. 2000, vol. 50, no. 1, pp. 27-46. Available from: https://doi.org/10.1016/S09396411(00)00090-4.

[9] MARMORAT, C., ARINSTEIN, A., KOIFMAN, N., TALMON, Y., ZUSSMAN, E., RAFAILOVICH, M. Cryo-imaging of hydrogels supermolecular structure. Scientific Reports. [online]. 2016, vol. 6, 25495. Available from: https://doi.org/10.1038/srep25495. 